\title{
ANÁLISE DA VIDA ÚTIL DE SERVIÇO DE ESTRUTURAS DE CONCRETO ARMADO SUJEITAS À CORROSÃO: UMA ABORDAGEM MECÂNICO- PROBABILÍSTICA
}

\author{
E. F. FELIX ${ }^{1}$, T. J. R. BALABUCH ${ }^{1}$, R. CARRAZEDO ${ }^{1}$, E. POSSAN ${ }^{2}$ \\ ${ }^{1}$ Departamento de Engenharia de Estruturas, Universidade de São Paulo (EESC/USP), São Carlos, Brasil. \\ ${ }^{2}$ Universidade Federal da Integração Latino-Americana (UNILA), Foz do Iguaçu, Brasil.
}

\section{RESUMO}

O presente trabalho apresenta uma análise mecânico-probabilística da vida útil de serviço de vigas de concreto sujeitas à corrosão por carbonatação. Os períodos de iniciação e propagação da corrosão são determinados por meio do cálculo de probabilidades de ocorrência dos estados limites de despassivação e fissuração, respectivamente. Os resultados encontrados apontam que a depender do tipo de cimento, do cobrimento, da taxa de armadura e do ambiente de exposição, a vida útil de serviço pode ser drasticamente reduzida, apresentando em muitos casos, confiabilidade estrutural e durabilidade inferior à requisitada em projeto. Ademais, os resultados demonstram a importância da consideração da aleatoriedade das variáveis, a fim de representar com maior fidelidade o processo de corrosão e, seus efeitos deletérios no concreto.

Palavras clave: Corrosão; vida útil de serviço; fissuração do concreto; confiabilidade; simulação de Monte Carlo.

\begin{abstract}
The present work presents a mechanical-probabilistic analysis of the service life of concrete beams subjected to corrosion due to carbonation. The initiation and propagation periods are determined by calculating the probability of occurrence of the limit states of dispassivation and cracking, respectively. The results show that depending on the type of cement, the concrete cover, the reinforcement ratio and the exposure environment, the service life can be drastically reduced, with in many cases, structural reliability and durability less than the required project. Moreover, the results demonstrate the importance of considering the randomness of the variables, in order to represent more accurately the corrosion process and its deleterious effects on the concrete.
\end{abstract}

Keywords: Corrosion; service life; cracking; reliability; Monte Carlo experiments.

\section{RESUMEN}

El presente trabajo presenta un análisis mecánico-probabilístico de la vida útil de servicio de vigas de hormigón sujetas a la corrosión por carbonatación. Los períodos de iniciación y propagación de la corrosión se determinan por medio del cálculo de probabilidades de ocurrencia de los estados límites de despassivación y fisuración, respectivamente. Los resultados encontrados apuntan que, a depender del tipo de cemento, del recubrimiento, de la tasa de armadura y del ambiente de exposición, la vida útil de servicio puede ser drásticamente reducida, presentando en muchos casos, confiabilidad estructural y durabilidad inferior a la requerida en proyecto. Además, los resultados demuestran la importancia de la consideración de la aleatoriedad de las variables, a fin de representar con mayor fidelidad el proceso de corrosión y, sus efectos deletéreos en el concreto.

Palabras clave: Corrosión; vida útil del servicio; fisuración del hormigón; confiabilidad; simulación de Monte Carlo. 


\section{INTRODUÇÃO}

A gestão da durabilidade da infraestrutura civil envolve gastos significativos e, em uma época de recursos públicos limitados, requer decisões difíceis para estabelecer prioridades de manutenção, reabilitação e substituição. Neste aspecto, percebe-se a importância do conceito de vida útil, que serve como base para uma abordagem holística de projeto (Ellingwood e Frangopol, 2016).

Uma das principais causas de redução da vida útil de estruturas de concreto armado é a corrosão, uma vez que esta envolve a perda de material da superfície do aço como resultado de uma ação química, e a perda material leva a redução de área efetiva na seção transversal e consequentemente a diminuição da capacidade de suportar cargas (Kari et al., 2014). No Brasil, por exemplo, a corrosão possuí um índice de ocorrência em estruturas de concreto que varia de 14 a $64 \%$ a depender da região de análise (Carmona e Marega, 1988; Dal Molin, 1988; Andrade, 1992).

A corrosão das armaduras de aço no concreto é um processo eletroquímico, causado pelas diferenças nas concentrações de íons dissolvidos, de maneira que parte do metal se torna catódica e outra anódica, resultando na perda de volume do material, gerando produtos de corrosão, e consequente redução de capacidade de suporte (Mehta e Monteiro, 2014; Geiker e Polder, 2016).

A formação dos produtos de corrosão gera um campo de tensões interno, localizado na zona de interface entre o aço e o concreto, que em função da sua magnitude leva a formação de fissuras que se propagam em direção às faces de concreto expostas à atmosfera (Kari et al., 2014; Nguyen et al., 2015).

De acordo com Helene (1986) a corrosão está, em geral, associada à carbonatação do concreto (corrosão uniforme) ou ao ingresso de cloretos (corrosão localizada). No que condiz o processo corrosivo iniciado pela carbonatação do concreto, o processo pode ser subdividido em duas fases, a iniciação (Figura 1a) e a propagação (Figura 1c), sendo a transição entre as duas fases caracterizada pela despassivação do aço (Figura 1b), momento em que a profundidade de difusão do $\mathrm{CO} 2$ (dd) é igual ao cobrimento da armadura (cl).

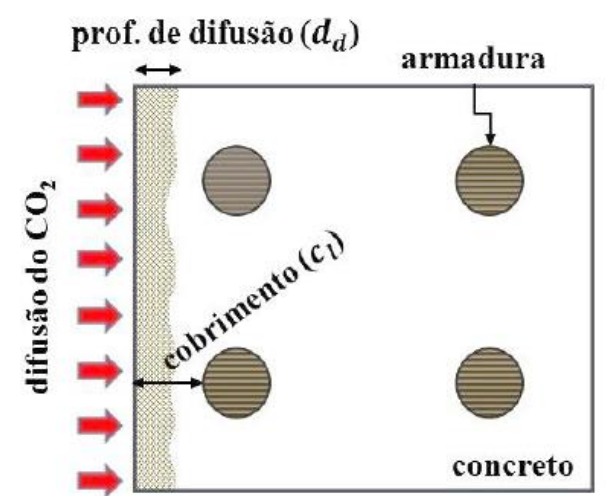

(I) Iniciação $\left(d_{d}<c_{l}\right)$

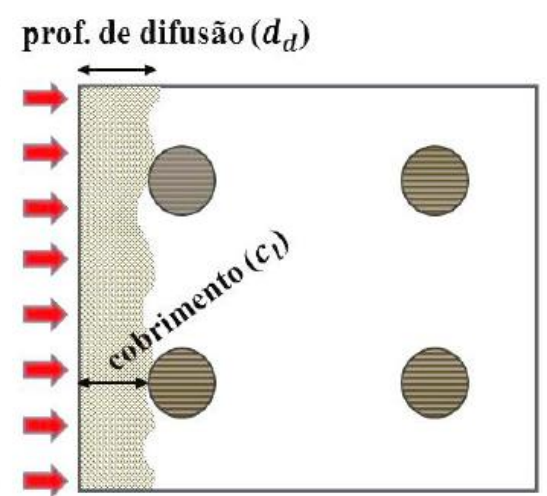

(II) Despassivação $\left(d_{d}=c_{l}\right)$

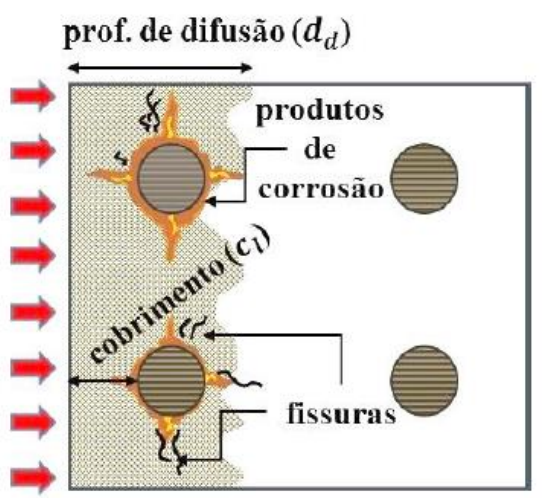

(III) Propagação $\left(d_{d}>c_{l}\right)$

Figura 1. Estágios do processo de corrosão por carbonatação no concreto.

A fase de iniciação (Figura 1a) se caracteriza pela difusão de diferentes agentes agressivos como o dióxido de carbono $(\mathrm{CO} 2)$ presentes no meio ambiente. O gás carbônico penetra na matriz porosa do concreto reagindo com os hidróxidos de cálcio $(\mathrm{Ca}(\mathrm{OH}) 2)$ da pasta de cimento, levando a formação do carbonato de cálcio (CaCO3). Este processo de carbonatação é responsável pela redução da porosidade e permeabilidade (Neville, 1997). Além disso, a carbonatação ocasiona na redução do pH do concreto (de 13 a aproximadamente 8), e consequentemente, na destruição da camada passivadora que envolve a armadura, deixando-a suscetível à corrosão (Chang e Chen, 2006). 
À medida que a frente de carbonatação atinge a profundidade do aço e promove sua despassivação, tem-se início a fase de propagação da corrosão. Até o instante de despassivação do aço, os danos não são perceptíveis, diferentemente do que ocorre durante o período de propagação (Tuutti, 1982; Possan, 2010; Köliö et al., 2017).

O período de propagação é caracterizado, de forma geral, pela taxa de corrosão e a qualidade do concreto para suportar as tensões internas geradas no processo de expansão do aço de vido a formação dos produtos de corrosão, também conhecido como óxido de férrico. O óxido férrico não hidratado (Fe2O3) tem um volume de cerca de duas vezes o do aço integro, sendo que ao hidratar-se, se expande ainda mais tornando-se poroso, aumentando o volume na interface aço-concreto de três a dez vezes, causando diversos danos ao concreto.

A expansão causada pela formação dos produtos de corrosão leva à fissuração do concreto, devido ao campo de tensões que é induzido na região de contato entre o aço e o concreto. Em função da magnitude das tensões de tração geradas pelos produtos de corrosão, macrofissuras surgem, facilitando o ingresso do $\mathrm{CO} 2$, e assim, aumentando o nível de degradação do concreto, afetando a durabilidade da estrutura (Broomfield, 2007; Köliö et al., 2017).

No que condiz a modelagem destes processos, boa parte das pesquisas se concentram no estudo do estágio de iniciação da corrosão, com o desenvolvimento de modelos para a estimativa da frente de carbonatação. Os primeiros modelos para a previsão da profundidade de carbonatação foram obtidos por meio de regressões linear e não linear, considerando diferentes fatores, por exemplo, a relação água/cimento $(\mathrm{a} / \mathrm{c})$, o tipo do aglomerante e as condições de exposição, como é visto nos trabalhos de Hamada (1968), Smolczyk (1968) e Helene (1986).

Nos anos seguintes, nos modelos desenvolvidos por Papadakis, Vayenas e Fardas (1991), Andrade (1992), Japsen (2002) e Hyverrt (2009), formulações físico-químicas referentes às reações de hidratação da pasta de cimento e da dissolução do $\mathrm{CO} 2$ na matriz porosa do concreto foram inseridas. No entanto, estes modelos possuem grande complexidade quanto à resolução das equações que regem a difusão do $\mathrm{CO} 2$ no concreto, além de possuírem parâmetros de difícil mensuração, por exemplo, o coeficiente de difusão do dióxido de carbono do concreto.

Quanto a modelagem do período de propagação da corrosão, pouca atenção era dada até meados da década de 80 em virtude das dificuldades em representar o avanço do fenômeno neste estágio e da reduzida quantidade de dados disponíveis na literatura. Porém com o surgimento e aprimoramento de técnicas numéricas, novas formulações foram desenvolvidas. De forma geral, os modelos que consideram o período de propagação, propostos ao longo dos últimos anos, podem ser classificados em empíricos (Cabrera, 1996; Almusallam et al., 1996; Du et al., 2005; Savija et al., 2013; Muthulingam e Rao, 2014), analíticos (Wang e Liu, 2006; Yuan e Ji, 2009; Shodja et al., 2010; Pengwei et al., 2011; Plucinski, 2015) e numéricos (Hansen e Saouma, 1999; Maruya et al., 2003; Du e Liu, 2014; Zhao et al., 2016).

Entretanto, um complicador natural da avaliação da corrosão é dado pela difusão do $\mathrm{CO} 2$ na matriz porosa do concreto. A difusão é controlada por complexas interações entre os mecanismos físicos e químicos dos materiais, onde várias fontes de incertezas podem ser observadas (Mehta e Monteiro, 2014). A penetração do gás carbônico é muitas vezes simplificada, sem perda significativa de generalidade, por problemas controlados apenas pelo processo de difusão. Além disso, existem as incertezas presentes nas propriedades dos materiais, nas condições ambientais e nos próprios parâmetros dos modelos matemáticos que simulam a corrosão, dificultando ainda mais as análises.

Diante disso, abordagens puramente determinísticas não são capazes de avaliar adequadamente a vida útil de estruturas em concreto armado sujeitas ao processo de corrosão, sendo necessário analisar o problema considerando a aleatoriedade dos diferentes parâmetros. 
Com os avanços da computação e com o advento da Teoria da Confiabilidade aplicada à análise de estruturas, a modelagem de fenômenos estruturais considerando as incertezas dos parâmetros tornouse muito atrativa, pois permitiu o tratamento dessas incertezas de uma forma teórica mais consistente através de associações estatísticas (Liberati et al., 2014). Sendo assim, de acordo com Liberati et al. (2014), o acoplamento de um modelo baseado em formulações matemáticas baseadas nas leis de difusão com algoritmos de confiabilidade pode resultar numa abordagem mais consistente, abrangente e confiável do que processos determinísticos. Tais modelos acoplados podem, por exemplo, estimar probabilidade de ocorrência do início do processo de corrosão, auxiliando a montagem de possíveis ações de inspeção e/ou manutenção programada em estruturas de concreto armado.

Diante do exposto, neste trabalho analisa-se a probabilidade de um elemento de concreto armado atingir o estado limite de serviço durante a vida útil de projeto (60 anos) a partir de uma abordagem combinada entre a teoria da confiabilidade e formulações matemáticas referentes aos processos de carbonatação, despassivação do aço, perda de seção útil da armadura e fissuração do concreto.

\section{MODELAGEM PROBABILÍSTICA DA VIDA ÚTIL DE SERVIÇO}

A fim de determinar a probabilidade de vigas de concreto armado sob corrosão uniforme atingir o estado limite de fissuração durante sua vida útil de projeto, período de 60 anos, de acordo com a NBR 6118 (ABNT, 2014), uma abordagem mecânica-probabilística via simulação de Monte Carlo foi considerada neste trabalho, de forma a inserir no processo de modelagem a aleatoriedade dos parâmetros envolvidos no fenômeno. Na sequência são apresentadas as formulações analíticas utilizadas para modelar o processo de difusão do $\mathrm{CO} 2$ no concreto, e estimar a abertura característica de fissuras. São apresentadas ainda, o acoplamento destas formulações à metodologia de análise probabilística de Monte Carlo.

\subsection{Descrição do modelo implementado para a análise da abertura de fissura}

Para a determinação da abertura característica de vigas de concreto armado, utilizou-se as formulações descritas na NBR 6118 (ABNT, 2014), às quais resumem-se em (1).

$$
w \leq\left\{\begin{array}{c}
w_{1}=\frac{\phi_{i}}{12,5 \cdot \lambda_{i}} \cdot \frac{\sigma_{s i}}{E_{s i}} \cdot \frac{3 \cdot \sigma_{s i}}{f_{c t m}} \\
w_{2}=\frac{\phi_{i}}{12,5 \cdot \lambda_{i}} \cdot \frac{\sigma_{s i}}{E_{s i}} \cdot\left(\frac{4}{\rho_{r i}}+45\right)
\end{array}\right.
$$

em que $w$ é a abertura de fissura característica $(\mathrm{mm}), \phi i$ é o diâmetro da armadura $i(\mathrm{~mm}), \lambda i$ é o coeficiente de conformação superficial da armadura (1,0 para barras lisas, 1,4 para barras dentadas e 2,5 para barras nervuradas), $\sigma s i$ é a tensão de tração da armadura $\left(\mathrm{kN} / \mathrm{cm}^{2}\right)$, Esi é o módulo de elasticidade da barra de aço $\left(\mathrm{kN} / \mathrm{cm}^{2}\right), f c t m$ é a tensão de tração média do concreto $\left(\mathrm{kN} / \mathrm{cm}^{2}\right)$ e $\rho r i$ é a taxa de armadura em relação à área envolvente.

Entretanto, como estamos interessados em analisar vigas de concreto susceptíveis à corrosão uniforme, o diâmetro da armadura, $\phi$, sofre um redução ao longo do tempo, sendo necessário determiná-la a cada instante de análise. Dessa forma, foram utilizadas formulações que fornecessem o diâmetro integro de aço (Stewart e Rosowsky, 1998) e da taxa de corrosão (Vu e Stewart, 2000) ao longo do tempo, dadas respectivamente em (2) e (3). 


$$
\begin{aligned}
& \phi\left(t_{p}\right)=\phi_{0}-\left(2 \cdot 0,0116 \cdot \eta \cdot t_{p}\right) \\
& \eta=\left(\frac{37,8 \cdot(1-a / c)^{-1,64}}{c o b}\right) \cdot 0,85 \cdot\left(t_{p}\right)^{-0,29}
\end{aligned}
$$

em que $\phi(t p)$ é o diâmetro integro de aço (em $\mathrm{mm}$ ) no tempo $t p$ (em anos) após iniciar o período de progressão da corrosão, calculado por (4), $\phi 0$ é o diâmetro inicial da barra (mm), $\eta$ representa a taxa de corrosão da armadura $\left(\mu \mathrm{A} / \mathrm{cm}^{2}\right), a / c$ é a relação água/cimento do concreto e cob é a espessura do concreto de cobrimento ( $\mathrm{mm})$.

$$
t_{p} \leq\left\{\begin{array}{c}
60-t_{\text {ini }} \quad \text { se } t_{\text {ini }}<60 \\
0 \text { se } t_{\text {ini }} \geq 60
\end{array}\right.
$$

em que tini é o instante de tempo em que ocorre a despassivação do aço. Destaca-se que neste trabalho, como o tempo de análise é de 60 anos, caso a despassivação ocorra após este período, o tempo de progressão é definido como nulo, conforme mostra a Equação (4).

Para a determinação do instante em que ocorre o início a degradação das armaduras pela corrosão e, consequentemente, a sua despassivação (tini), acoplou-se à (4) um modelo analítico da profundidade de carbonatação do concreto proposto por Possan (2010), o qual possibilitou a determinação do tempo em que a profundidade de carbonatação é superior ao cobrimento da viga (c), conforme apresentado em (5).

$$
t_{i n i}=20\left[\frac{c}{k_{c}\left(\frac{20}{f_{c k}}\right)^{k f c} e^{\left[\left(\frac{K_{a d} a d^{1.5}}{40+f_{c k}}\right)+\left(\frac{K_{c o 2} C 2^{0.5}}{60+f_{c k}}\right)-\left(\frac{K_{U R}(0.58-U R)^{1.5}}{100+f_{c k}}\right)\right]} K_{c e}}\right]^{2}
$$

em que c é o cobrimento da viga de concreto $(\mathrm{mm})$, fc é a resistência característica à compressão axial do concreto (MPa), kc é o fator variável referente ao tipo de cimento empregado (Tabela 1), kfc é o fator variável referente à resistência à compressão axial do concreto, em função do tipo de cimento utilizado (Tabela 1), t é a idade do concreto (em anos), ad é o teor de adição pozolânica no concreto (em \% em relação à massa de cimento), kad é o fator variável referente às adições pozolânicas do concreto - sílica ativa, metacaulim e cinza de casca de arroz, em função do tipo de cimento utilizado (Tabela 1), UR é a umidade relativa média, em $(\% * 0,01)$, kur é o fator variável referente à umidade relativa, em função do tipo de cimento utilizado (Tabela 1), $\mathrm{CO} 2$ é o teor de $\mathrm{CO} 2$ da atmosfera (\%), $\mathrm{kCO} 2$ é o fator variável referente ao teor de $\mathrm{CO} 2$ do ambiente, em função do tipo de cimento utilizado (Tabela 1), kce é o fator variável referente à exposição à chuva, em função das condições de exposição da estrutura (Tabela 2). 
Tabela 1. Coeficientes em função: das características do concreto e das condições ambientais. (Possan, 2010).

\begin{tabular}{|c|c|c|c|c|c|}
\hline \multirow{2}{*}{ Tipo de Cimento } & \multicolumn{3}{|c|}{ Características do concreto } & \multicolumn{2}{c|}{ Condições ambientais } \\
\cline { 2 - 5 } & kc & kfc & kad & kco2 & Kur \\
\hline CP I & 19,80 & 1,70 & 0,24 & 18,00 & 1300 \\
\hline CP II E & 22,48 & 1,50 & 0,32 & 15,50 & 1300 \\
\hline CP II F & 21,68 & 1,50 & 0,24 & 18,00 & 1100 \\
\hline CP II Z & 23,66 & 1,50 & 0,32 & 15,50 & 1300 \\
\hline CP III & 30,50 & 1,70 & 0,32 & 15,50 & 1300 \\
\hline CP IV & 33,27 & 1,70 & 0,32 & 15,50 & 1000 \\
\hline CP V ARI & 19,80 & 1,70 & 0,24 & 18,00 & 1300 \\
\hline
\end{tabular}

Tabela 2. Coeficientes do modelo em função das condições de exposição. (Possan, 2010).

\begin{tabular}{|c|c|}
\hline \multicolumn{2}{|c|}{ Condições de exposição da estrutura } \\
\hline Proteção à chuva & kce \\
\hline Ambiente interno Protegido da chuva & 1,30 \\
\hline Ambiente externo Protegido da chuva & 1,00 \\
\hline Ambiente externo Desprotegido da chuva & 0,65 \\
\hline
\end{tabular}

Para a determinação da tensão atuante nas armaduras, utilizou-se as equações referentes ao dimensionamento à flexão de vigas de concreto armado, descritas na NBR 6118 (ABNT, 2014). Diante da consideração que as vigas analisadas neste trabalho são bi-apoiadas e com carregamento linearmente distribuído, a tensão de tração que atua em uma barra longitudinal é dada por (6).

$$
\sigma_{s i}=\frac{\left[\frac{g l^{2}}{8}+0.4\left(\frac{q l^{2}}{8}\right)\right]}{0,8\left(h-\left(0.5 \phi_{i}+c+\phi_{t}\right)\right) A_{s i}}
$$

em que h é a altura da seção transversal da viga $(\mathrm{m})$, g é a carga permanente (em $\mathrm{kN} / \mathrm{m})$, q é a carga acidental (em $\mathrm{kN} / \mathrm{m}), 1$ é o comprimento da viga bi-apoiada (em $\mathrm{m})$ e c, é o cobrimento, valor que define em (5) a profundidade de carbonatação que leva à despassivação do aço.

\subsection{Análise probabilística via simulação de Monte Carlo}

A fim de realizar análises probabilísticas de vigas de concreto armado atingirem o estado limite último de fissuração ao longo da vida útil de projeto, as formulações anteriormente descritas, foram acopladas e analisadas para diferentes tipos de cimento e ambientes de exposição, por meio de uma abordagem via teoria da confiabilidade. As modelagens e análises foram realizadas por meio da simulação de Monte Carlo.

O método de simulação de Monte Carlo é um procedimento numérico, amplamente utilizado em diversas áreas, para a realização de experimentos aleatórios em problemas de confiabilidade. Neste método, amostras de variáveis aleatórias são utilizadas para construir um conjunto de realizações do experimento com o intuito de descrever a falha, os espaços seguros, correlacionados à confiabilidade, e calcular a probabilidade do evento, conforme (7).

As amostras utilizadas para o cálculo da probabilidade de falha, são construídas com base na distribuição estatística atribuída a cada variável aleatória do problema. Como o método está baseado na simulação da função de estado limite, quanto maior for a amostra gerada, mais precisa será a descrição 
do espaço e mais precisa será a probabilidade de falha ou confiabilidade determinada (Beck, 2018).

$$
P_{f}=\int_{G \leq 0} f_{x}\left(x_{1}, x_{2}, \ldots, x_{n}\right) d x_{1}, d x_{2}, \ldots, d x_{n}
$$

em que $f x(X)$ é a função conjunta de densidade das variáveis $X$.

Como a avaliação da integral definida em (7) é quase impossível na prática, procedimentos alternativos foram desenvolvidos com base no conceito de índice de confiabilidade $\beta$ (Beck, 2018). O índice de confiabilidade é definido como a distância entre o ponto médio e o ponto de falha alocado sobre a função de estado limite, $G(X)=0$.

De forma geral, o método consiste na construção de uma amostra para as variáveis aleatórias envolvidas no problema a ser simulado, e assim, a probabilidade de falha é calculada utilizando um estimador, baseado na avaliação da função de estado limite, de acordo como apresentado em (8). O estimador I(xi), baseado na função de estado limite, é dado em (9), sendo seu valor 0 ou 1, sendo 0 para o caso em que não há falha e, 1, para quando há falha.

$$
\begin{aligned}
& P_{f}=\int_{G \leq 0} f_{x}\left(x_{i}\right) d x_{i}=\int_{G \leq 0} I\left(x_{i}\right) f_{x}\left(x_{i}\right) d x_{i}=E\left[I\left(x_{i}\right)\right] \\
& I\left(x_{i}\right)= \begin{cases}1, & G \leq 0 \\
0, & G>0\end{cases}
\end{aligned}
$$

Simulando a função de estado limite para um número conveniente de amostras, o valor médio I(xi) será uma estimativa para a probabilidade de falha, assim, de acordo com (10) a probabilidade de falha pode ser facilmente estimada para todo o conjunto de amostras.

$$
\overline{P_{f}}=E\left[I\left(x_{i}\right)\right]=\frac{1}{N} \sum_{i=1}^{N} I\left(x_{i}\right)
$$

em que $N$ é o número de simulações, isto é, de avaliações da equação de estado limite do problema analisado.

Por fim, definido o método de estimativa da probabilidade de falha, a equação de estado limite a ser analisada neste trabalho é dada em (11) e, refere-se ao evento da viga atingir o estado limite de serviço durante a vida útil de projeto.

$$
g(x)=w_{c}-w
$$

em que $w c$ é a abertura crítica da fissura (em mm), e $w$, é a abertura característica de fissuração do concreto (em mm), calculada por (1). A abertura crítica do concreto é dada em função do ambiente de exposição e da sua agressividade, conforme descrito na NBR 6118 (ABNT,2014).

De acordo com a expressão apresentada em (11), tem-se que falhar do evento ocorre quando $g \leq 0$, e não falha quando $g>0$. Assim, em nossas análises, a falha ocorre quando a abertura característica de fissuração for igual ou superior à abertura crítica da fissura. Os demais casos representam a não falha do evento. 


\subsection{Descrição das estruturas analisadas}

A estrutura analisada neste trabalho consiste em vigas de concreto armado dimensionadas de acordo com os procedimentos da NBR 6118 (ABNT, 2014). O esquema que representa o carregamento utilizado para o dimensionamento no estado limite último (ELU) e para a análise do estado limite de serviço (ELS) são apresentados na Figura 2, assim como os detalhes construtivos da mesma.

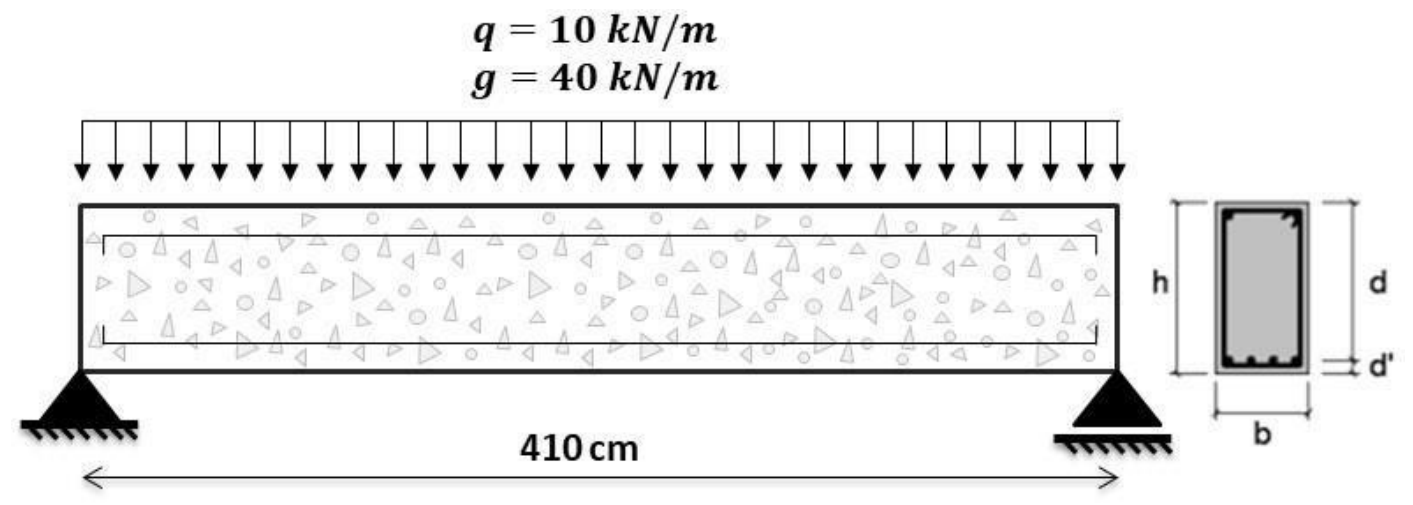

Figura 2. Detalhamento simplificado da viga de concreto armado.

Para a definição das vigas e as simulações, as variáveis foram caracterizadas como determinísticas ou aleatórias. As variáveis determinísticas foram divididas em variáveis com valores fixos e não fixos, sendo as variáveis com valores fixos: altura de $40 \mathrm{~cm}$; largura da seção transversal de $22 \mathrm{~cm}$; vão livre de $410 \mathrm{~cm}$; estribos com 0,63 cm de diâmetro; barras longitudinais com $2 \mathrm{~cm}$ de diâmetro; concretos produzidos com relação água cimento de 0,4 ; resistência à tração do concreto de $0,21 \mathrm{kN} / \mathrm{cm}^{2}$; coeficiente de Poisson do concreto de 0,2; aço CA-50; módulo de elasticidade do aço de 21000 $\mathrm{kN} / \mathrm{cm}^{2}$; resistência à tração do aço de $50 \mathrm{kN} / \mathrm{cm}^{2}$.

As variáveis determinísticas com valores não fixos, foram definidas com o propósito de analisar a sua influência no processo de degradação das vigas, sendo: kc, kfc, kad, kur, $\mathrm{kCO} 2$ (dependentes do tipo de cimento, de acordo com a Tabela 1), e kce (dependente do tipo de exposição à chuva, ver de acordo com a Tabela 2).

Tabela 3. Dados referentes ao concreto e ambiente de exposição.

\begin{tabular}{|c|c|c|c|}
\hline Variável & Média & $\begin{array}{c}\text { Coeficiente de } \\
\text { variação }\end{array}$ & Distribuição \\
\hline Cobrimento (mm) & 30 & $10 \%$ & Normal \\
\hline $\begin{array}{c}\text { Resistência à compressão do } \\
\text { concreto (MPa) }\end{array}$ & 25 & $10 \%$ & Normal \\
\hline Umidade relativa (\%) & 70 & $10 \%$ & Normal \\
\hline Teor de CO2 (\%) & 0,01 & $15 \%$ & Normal \\
\hline
\end{tabular}

No que diz respeito às variáveis aleatórias, neste trabalho é analisada a variabilidade presente em quatro parâmetros relacionados à corrosão e à fissuração do concreto: (i) o cobrimento das armaduras; (ii) a resistência à compressão do concreto; (iii) a umidade relativa e; (iv) o teor de $\mathrm{CO} 2$ no ambiente. A Tabela 3 apresenta as quatro variáveis aleatórias com suas respectivas médias, coeficiente de variação e função de distribuição. 


\section{RESULTADOS}

Inicialmente, na Figura 3 é apresentado o avanço da profundidade de carbonatação do concreto e a área útil de aço para os seis tipos de cimento Portland (cimentos comercializados no Brasil, de acordo com a Tabela 1), e os diferentes ambientes de exposição simulados (Ambiente interno protegido da chuva e externo desprotegido da chuva). Os resultados foram obtidos considerando os valores médios das variáveis aleatórias do problema, conforme Tabela 3.
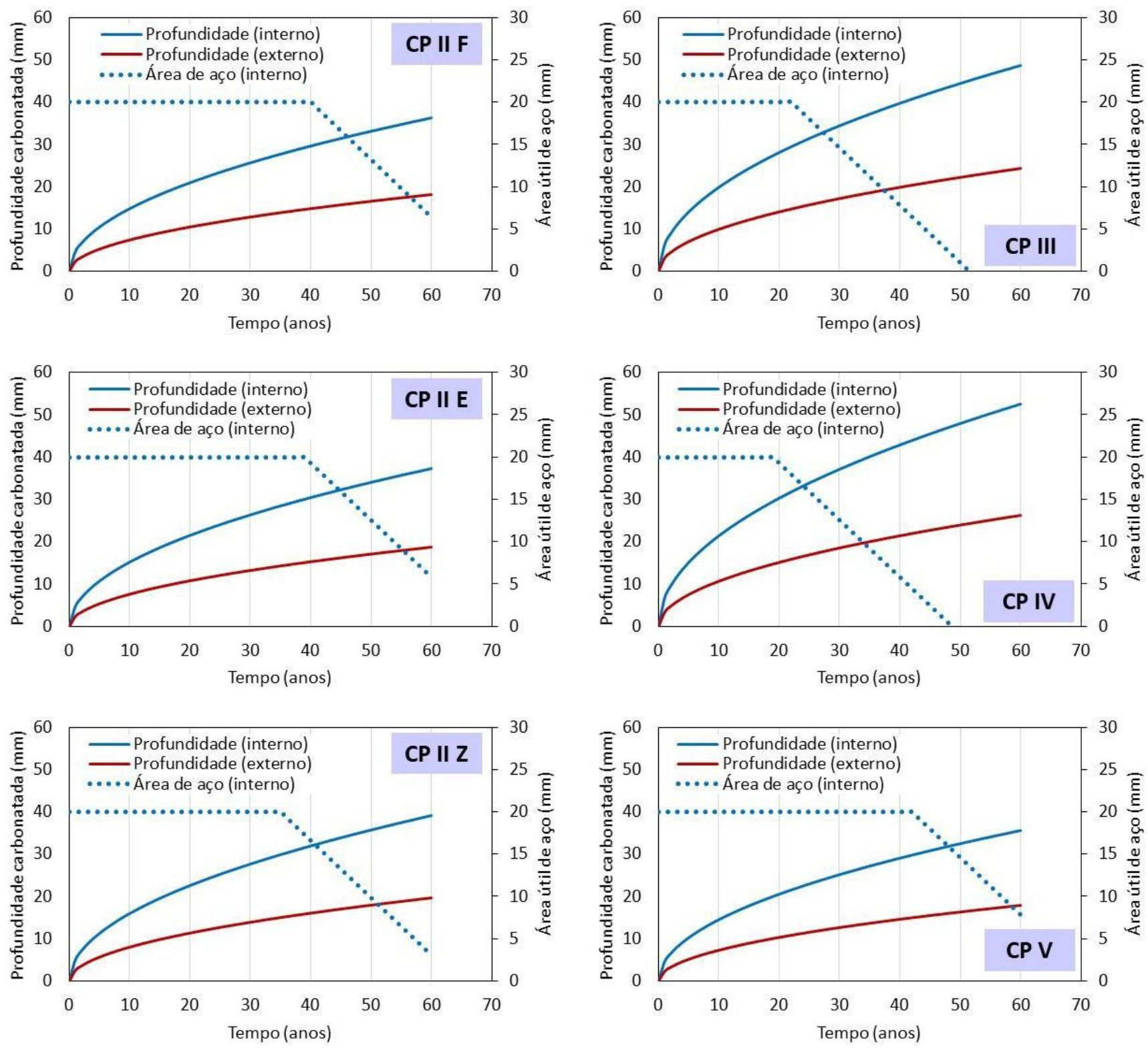

Figura 3. Avanço da carbonatação no concreto e área útil de aço.

Observa-se na Figura 3 que durante a fase de iniciação da corrosão a área útil de aço das armaduras não é alterada, e que, a partir do momento em que ocorre a despassivação da armadura, a perda de seção acontece de forma continua e linear, diante da consideração que nenhuma manutenção ou reparo é realizado no elemento estrutural, conforme observado por Vu e Stewart (2000). Os autores observaram por meio de experimentos que a perda de seção de aço ocorre com maior intensidade nos primeiros 
anos da manifestação patológica, e que, se o processo de degradação não é cessado, a perda de seção útil de aço pode chegar a $50 \%$ em 10 anos.

Dando continuidade na análise dos resultados, é possível observar por meio da Figura 3, que para os cenários simulados neste trabalho, e considerando a análise determinística, não ocorre a

despassivação do aço para o ambiente externo desprotegido da chuva, motivo pela qual não existem curvas referente a perda de aço para cenário.

A seguir, na Figura 4, são apresentadas as probabilidades de os elementos de concreto atingirem o estado limite de serviço durante o período que compreende a vida útil de projeto. Para a análise do estado limite de serviço, considerou-se o estado limite de abertura de fissura, $\mathrm{O}$ valor crítico de abertura de fissura adotado foi $w c=0,3 \mathrm{~mm}$, conforme prescreve a NBR 6118 (ABNT, 2014) para estruturas de concreto com classe de agressividade ambiental II (agressividade média). A determinação das probabilidades foi realizada mediante simulação de Monte Carlo, já descrito neste trabalho. Ao todo foram geradas 50.000 amostras para a realização das simulações e cálculo de probabilidades.
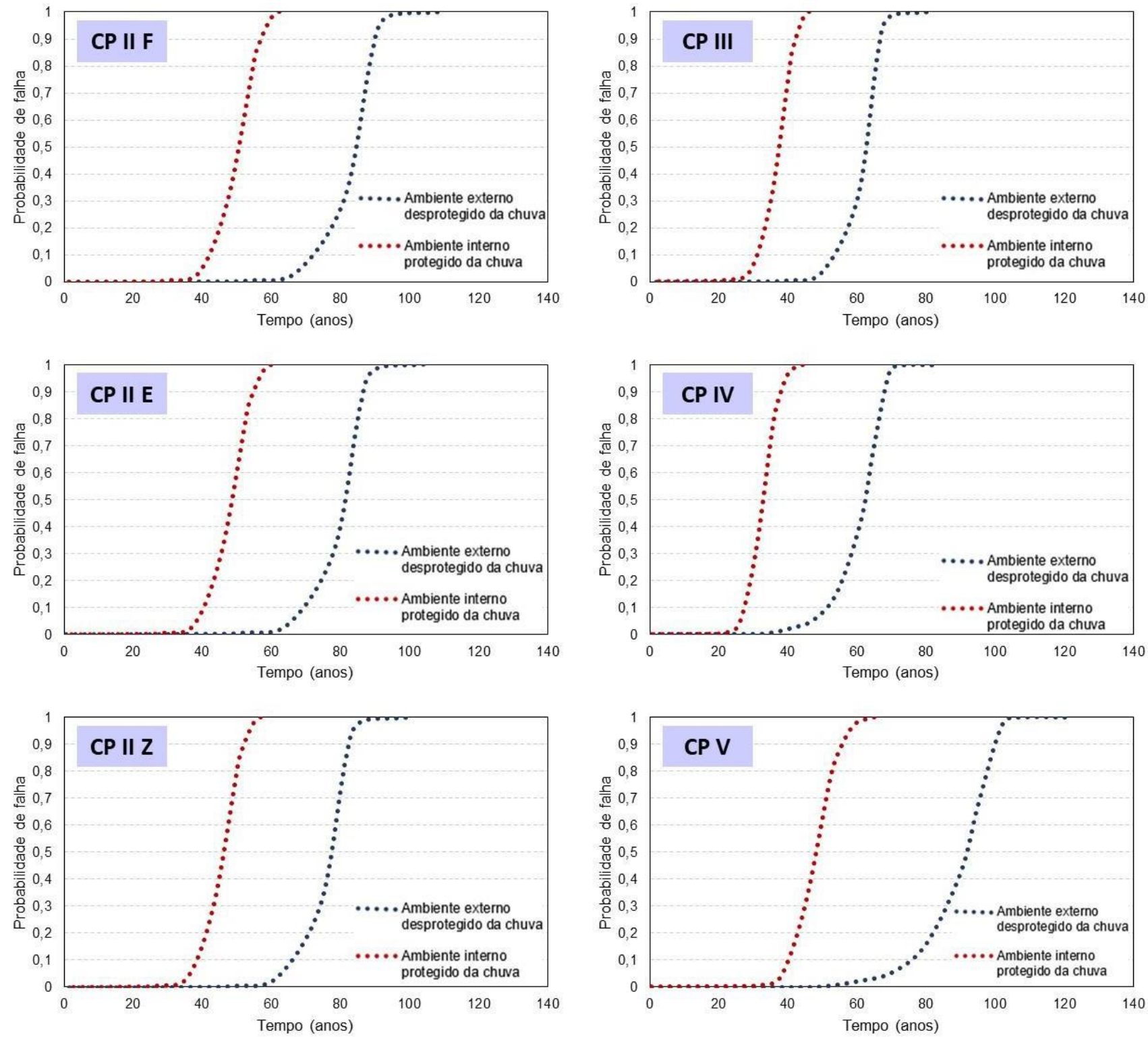

Figura 4: Probabilidade de os elementos atingirem o estado limite de serviço. 
A partir dos resultados apresentados na Figura 4, verificou-se que a probabilidade de falha, que neste trabalho refere-se ao elemento alcançar fissuras com aberturas superiores à crítica, é nula para os 20 primeiros anos de vida, independentemente do tipo de cimento e do ambiente de exposição. Observase também que a partir do momento em que ocorre a despassivação, as probabilidades de falha aumentam drasticamente para todos os casos considerados no estudo, e que, as estruturas de concreto atingem o estado limite último de fissuração durante o período de vida útil de projeto para todos os casos, com exceção, do elemento de concreto produzido com cimento CP II F e em ambiente desprotegido da chuva.

Na Figura 4 é possível observar ainda que, o ambiente de exposição interno protegido da chuva, apresenta condições mais agravante que o ambiente externo desprotegido, e que a depender do tipo de cimento utilizado na produção do concreto, a probabilidade de falha para um período de vida do elemento, é significativamente maior, demonstrando a importância de se conhecer corretamente o ambiente de exposição do concreto ainda na fase de projeto, e selecionar o tipo de cimento que proporciona maio durabilidade ao componente estrutural.

\section{CONCLUSÕES}

Diante dos resultados obtidos com as análises determinísticas e probabilísticas, pode-se concluir que:

- Um cobrimento adequado garante não só um período maior para levar à despassivação da armadura como também para atingir o limite de abertura de fissuras;

- Se apenas considerada a perda de seção da armadura como consequência da corrosão, as fissuras do concreto alcançam dimensões superiores às recomendadas pela NBR 6118 (ABNT, 2014) antes que a estrutura entre na metade de sua vida útil de projeto, principalmente para os concretos com cimento CP II E, CP II F, CP II Z e CP III;

- Concretos produzidos com cimentos de maior durabilidade, por exemplo, CP V, apresentam maior resistência à carbonatação e, consequentemente, à corrosão, o que leva nestes casos a índices de confiabilidade maiores (menores probabilidades de atingir o estado limite de fissuras dentro da vida útil de projeto);

- Diante das análises, observou-se que das quatro variáveis aleatórias utilizadas, a UR e o FCK, foram as que apresentaram maior relevância e sensibilidade frente à probabilidade de a viga atingir o estado limite último de fissuração durante sua vida útil;

- Quanto à análise da proteção da estrutura à chuva, observou-se que a viga, independente do cimento utilizado em sua produção, possuí maior probabilidade de alcançar o estado limite último de fissuração quando situada em ambiente interno protegido da chuva, e mais, que essa probabilidade é significativamente maior que a da viga em ambiente externo desprotegido.

Assim, considerando os resultados obtidos neste trabalho, a durabilidade de estruturas de concreto armado deve ser associada a valores mais rigorosos de espessura de cobrimento, bem como concretos menos permeáveis e uma melhor análise do futuro ambiente de exposição para que um correto tipo de cimento seja adotado e potencialize a durabilidade do componente estrutural. O maior controle na manutenção da espessura da camada de concreto do cobrimento especificada no projeto garante menor variabilidade da mesma e, consequentemente, diminui a probabilidade de ocorrência do início da corrosão.

Acredita-se, ainda, que a abordagem probabilística do problema da vida útil através da consideração da aleatoriedade das variáveis envolvidas demonstrou-se adequada para uma determinação mais realística dos tempos de iniciação e progressão da corrosão, fornecendo assim, informações necessárias e importantes para a criação de programas de inspeção e manutenção da estrutura no decorrer de sua vida. Ademais, os resultados apontam a aplicabilidade da metodologia adotada para tratar a aleatoriedade das variáveis de problemas correlatos à área de patologias. 


\section{AGRADECIMENTOS}

À Coordenação de Aperfeiçoamento Pessoal de Nível Superior (CAPES) e ao Conselho Nacional de Desenvolvimento Científico e Tecnológico (CNPq-141078/2018-9) pelo fomento oferecido à pesquisa.

\section{REFERÊNCIAS}

Almusallam, A. A., Al-Gahtani, A. S., Aziz, A. R., Rasheeduzzafar, R. (1996), Effect of reinforcement corrosion on bond strength. Construction and Building Materials. 10(2):123-129.

Andrade, C. (1992), "Manual para diagnóstico de obras deterioradas por corrosão de armaduras". Tradução de Antônio Carmona e Paulo Helene, São Paulo: PINI, p. 104.

Associação Brasileira de Normas Técnicas. (2014), NBR 6118: Projeto de estruturas de concreto. Rio de Janeiro.

Beck, A. T. (2019), “Confiabilidade e segurança das estruturas”. São Paulo: Elsevier Campus, p. 448. Broomfield, J.P. (2007), “Corrosion of steel in concrete: understanding, investigation and repair”. 2.ed. New York, Taylor \& Francis, p. 294.

Cabrera, J. G. (1996), Deterioration of Concrete Due to Reinforcement Steel Corrosion. Cement \& Concrete Composites. 18:47-59.

Carmona, A. F., Marega, A. (1988), "Retrospectiva da patologia no Brasil: Estudo Estatístico", In: Jornadas em Español y Portugués sobre Estructuras y Materiales, CEDEX, IETcc, 1988, pp. 325-348. Chang, C. F., Chen, J. W. (2006), The experimental investigation of concrete carbonation depth. Cement and Concrete Research. 36:1760-1767.

Dal Molin, D. C. C. (1988), "Fissuras em estruturas de concreto armado: análise das manifestações típicas e levantamento de casos ocorridos no Estado do Rio Grande do Sul", Masters Thesis, Universidade Federal do Rio Grande do Sul, Porto Alegre.

Du, Y. G., Clark, L. A., Chan, A. H. C. (2005), Residual Capacity of corroded reinforcing bars. Magazine of Concrete Research. 57(3):135-147.

Du, X., Liu, J. (2014), Meso-scale numerical investigation on cracking of cover concrete induced by corrosion of reinforcing steel. Engineering Failure Analysis. 39:21-33.

Ellingwood, B. R., Frangopol, D. M. (2016), Introduction to the State of the Art Collection: Risk-Based Lifecycle Performance of Structural Systems. Journal of Structural Engineering. 142:1-15. Geiker, M. R., Polder, R. B. (2016), Experimental support for new electro active repair method for reinforced concrete. Materials and Corrosion. 67:600-606.

Hamada, M. (1969), "Neutralization (carbonation) of concrete and corrosion of reinforcing steel", In: International Symposium on the Chemistry of Cement, 5, 1969. Tokyo. Proceedings... Tokyo, 1969, Part. III, v. II/4, p.343-369.

Hansen, E. J., Saouma, V. E. (1999), Numerical simulation of reinforced concrete deterioration: Part II - Steel corrosion and concrete cracking. ACI Materials Journal. 96(2):173-180.

Helene, P. R. L. (1986), "Corrosão em Armaduras para Concreto Armado”. São Paulo: IPT, PINI, 1986.

Kari, O. P., Puttonen, J., Skantz, E. (2014), Reactive transport modelling of long-term carbonation. Cement and Concrete Composites. 52:42-53.

Köliö, A., Pakkala, T. A., Hohti, H., Laukkarinen, A., Lahdensivu, J., Mattila, J., Pentti, M. (2017), The corrosion rate in reinforced concrete facades exposed to outdoor environment. Materials and Structures. 50:1-16.

Liberati, E. A. P., Leonel, E. D., Nogueira, C. G. (2014), Influence of the reinforcement corrosion on the bending moment capacity of reinforced concrete beams: a structural reliability approach. Ibracon Structures and Materials Journal. 7(3):379-413. 
Maruya, T., Hsu, K., Takeda, H., Tangtermsirikul, S. (2003), Numerical Modeling of Steel Corrosion in Concrete Structures due to Chloride Ion, Oxygen and Water Movement. Journal of Advanced Concrete Technology. 1(2):147-160.

Mehta, P. K., Monteiro, P. J. M. (2014), “Concreto: Microestrutura, Propriedades e Materiais”. 2.ed. São Paulo, IBRACON, p.751.

Muthulingam, S., Rao, B. N. (2014), Non-uniform time-to-corrosion initiation in steel reinforced concrete under chloride environment. Corrosion Science. 82:304-315.

Neville, A. M. (1997), “Propriedades do concreto”, São Paulo: PINI, p. 828.

Nguyen, T. T. H., Bary, B., De Larrard, T. (2015), Coupled carbonation-rust formation damage modeling and simulation of steel corrosion in 3D mesoscale reinforced concrete. Cement and Concrete Research. 74:95-107.

Papadakis, V. G., Vayenas, C. G., Fardis, M. N. (1991), Fundamental Modeling andExperimental Investigation of Concrete Carbonation. ACI Materials Journal. 88(4).

Possan E. (2010), "Modelagem da carbonatação e previsão de vida útil de estruturas de concreto em meio urbano”, Tese de Doutorado em Engenharia, Programa de Pós-Graduação em Engenharia Civil, Universidade Federal do Rio Grande do Sul, Porto Alegre.

Pengwei, X., Ning, Y., Linwang, S., Guobao, L., Lebin, W. (2011), Finite Element Analysis of Expansive Behaviour due to Reinforcement Corrosion in RC Structure. Procedia Engineering. 12:117126.

Plucinski, P. (2015), Numerical analysis of stress distribution in concrete around corroding rebar. Procedia Engineering. 108:598-607.

Rao, A. S., Lepech, M. D., Kiremidjian, A. S., Sun, X. Y. (2017), Simplified structural deterioration model for reinforced concrete bridge piers under cyclic loading. Structure and Infrastructure Engineering. 13:55-66.

Savija, B., Lukovic, M., Pacheco, J., Schlangen, E. (2013), Cracking of the concrete cover due to reinforcement corrosion: A two-dimensional lattice model study. Construction and Building Materials. 22:626-638.

Shodja, H. M., Kiani, K., Hashemian, A., A. (2010), Model for the evolution of concrete deterioration due to reinforcement corrosion. Mathematical and Computer Modelling. 52:1403-1422.

Smolczyk, H. G. (1969), “Written Discussion”, In: The International Symposium on the Chemistry of Cement, V. Tokyo, 1969. Proceedings... Tokyo, 1969, Part III, v. II/4, p.369-384.

Stewart, M. G., Rosowsky, D. V. (1998), Structural safety and serviceability of concrete bridges subject to corrosion. Journal of Infrastructure Systems. 4:146-155.

Tuutti, K. (1982), "Corrosion of steel in concrete”. Stockholm, Swedish Cement and Concrete Research Institute.

Vu, K. A. T., Stewart, M. G. (2000), Structural reliability of concrete bridges including improved chloride-induced corrosion models. Structural Safety. 22:313-333.

Wang, X., Liu, X. (2006), Bond strength modelling for corroded reinforcements. Construction and Building Materials. 20:177-186.

Yuan, Y., Ji, Y. (2009), Modeling corroded section configuration of steel bar in concrete structure. Construction and Building Materials. 23:2461-2466.

Zhao, Y., Dong, J., Wu, Y., Jin, W. (2016), Corrosion-induced concrete cracking model considering corrosion product-filled paste at the concrete/steel interface. Construction and Building Materials. 116:273-280. 